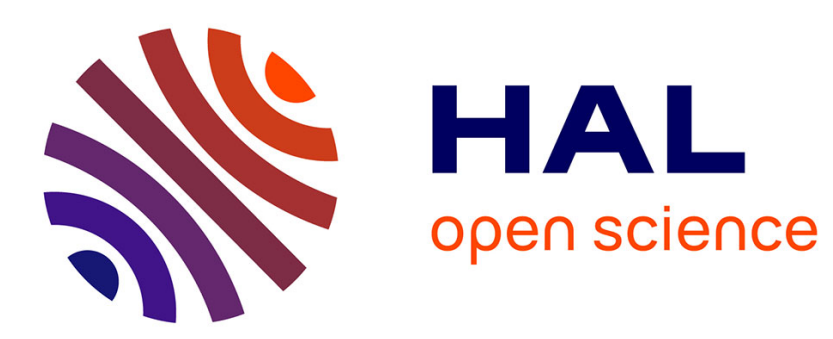

\title{
Quantum Dynamics of Atomic Magnets: Cotunneling and Dipolar-Biased Tunneling
}

R. Giraud, W. Wernsdorfer, A. M. Tkachuk, D. Mailly, B. Barbara

\section{To cite this version:}

R. Giraud, W. Wernsdorfer, A. M. Tkachuk, D. Mailly, B. Barbara. Quantum Dynamics of Atomic Magnets: Cotunneling and Dipolar-Biased Tunneling. Physical Review Letters, 2003, 91 (25), pp.257204. 10.1103/PhysRevLett.91.257204 . hal-01656622

\section{HAL Id: hal-01656622 \\ https://hal.science/hal-01656622}

Submitted on 5 Dec 2017

HAL is a multi-disciplinary open access archive for the deposit and dissemination of scientific research documents, whether they are published or not. The documents may come from teaching and research institutions in France or abroad, or from public or private research centers.
L'archive ouverte pluridisciplinaire HAL, est destinée au dépôt et à la diffusion de documents scientifiques de niveau recherche, publiés ou non, émanant des établissements d'enseignement et de recherche français ou étrangers, des laboratoires publics ou privés. 


\title{
Nuclear Spin Driven Quantum Relaxation in $\mathrm{LiY}_{0.998} \mathrm{Ho}_{0.002} \mathrm{~F}_{4}$
}

\author{
R. Giraud, ${ }^{1}$ W. Wernsdorfer, ${ }^{1}$ A. M. Tkachuk,${ }^{2}$ D. Mailly, ${ }^{3}$ and B. Barbara ${ }^{1}$ \\ ${ }^{1}$ Laboratoire de Magnétisme Louis Néel, CNRS, BP166, 38042 Grenoble Cedex-09, France \\ ${ }^{2}$ All-Russia Scientific Center "S. I. Vavilov State Optical Institute," 199034 St. Petersburg, Russia \\ ${ }^{3}$ Laboratoire de Photonique et de Nanostructures, CNRS, 196 Avenue H. Ravera, 92220 Bagneux, France
}

(Received 12 February 2001; published 17 July 2001)

\begin{abstract}
Staircaselike hysteresis loops of the magnetization of a $\mathrm{LiY}_{0.998} \mathrm{Ho}_{0.002} \mathrm{~F}_{4}$ single crystal are observed at subkelvin temperatures and low field sweep rates. This behavior results from quantum dynamics at avoided level crossings of the energy spectrum of single $\mathrm{Ho}^{3+}$ ions in the presence of hyperfine interactions. Enhanced quantum relaxation in constant transverse fields allows the study of the relative magnitude of tunnel splittings. At faster sweep rates, nonequilibrated spin-phonon and spin-spin transitions, mediated by weak dipolar interactions, lead to magnetization oscillations and additional steps.
\end{abstract}

DOI: $10.1103 /$ PhysRevLett.87.057203

The problem of quantum dynamics of a two-level system coupled to an environment (boson or fermion bath) is at the core of mesoscopic physics [1]. We show that the new field of "mesoscopic magnetism," which studies the tunneling of large magnetic moments in the presence of phonons and spins, is not limited to molecular complexes and nanoparticles, but it can be extended to other systems such as rare-earth ions. After the first studies on large spin molecules $\mathrm{Mn}_{12}$-ac [2,3] and $\mathrm{Fe}_{8}$ [4], the role of the spin bath on the tunnel mechanism was shown [5-9]. In particular, quasistatic fields due to dipolar interactions between molecules lead to a distribution of internal fields, and field fluctuations, essentially of nuclear spins, give homogeneous level broadening allowing the restoration of tunneling in a finite energy window, at low temperature; this broadening being much larger than the phonon one, it is more relevant to induce tunneling. This mechanism is efficient unless all nuclear spins of the molecule are frozen, which occurs only below the $\mathrm{mK}$ scale. In low spin molecules, large tunneling gaps favor spin-phonon transitions. Although the hyperfine induced level broadening is the same as in large spin molecules, the phonon bath becomes as important as the spin bath [10]. In all these cases, the role of field fluctuations was clearly evidenced.

This description is for the relatively weak hyperfine interactions of $\mathrm{Mn}_{12}$ or $\mathrm{Fe}_{8}$ molecules, and therefore for incoherent nuclear spin fluctuations. The question as to what really happens when an electronic moment tunnels, while it is strongly coupled to its nuclear spin, has not yet a clear answer. Contrary to the $3 d$ group, hyperfine interactions are very large in $4 f$ elements. Diluted rare-earth ions in a nonmagnetic insulating single crystal are therefore very suitable to study the possible entanglement of nuclear and electronic moments, when tunneling occurs. Our choice was the weakly doped rare-earth fluoride series $\mathrm{LiY}_{1-x} \mathrm{R}_{x} \mathrm{~F}_{4}$, in which high quality single crystals are mainly investigated for applications in high-power laser diodes [11]. Note that EPR spin echo of magnetic tunneling states has already been observed in a $1 \%$ Dy-doped crystal [12]. At higher concentrations, these crystals were
PACS numbers: $75.45 .+\mathrm{j}, 71.70 . J p, 76.30 . \mathrm{Kg}$

used for phase transition studies of dipolar ordered magnets [13]. Among them, the holmium doped fluoride is a random, dipolar coupled system with an Ising ground state doublet $\left(g_{\text {eff }} \approx 13.3\right.$ [14]; see also [15] and references therein) and a pure isotope $I=7 / 2$ nuclear spin. The magnetic properties of the Ising ferromagnet $\mathrm{LiHoF}_{4}$ and spin glass $\mathrm{LiY}_{0.833} \mathrm{Ho}_{0.167} \mathrm{~F}_{4}$ have been studied by ac susceptibility [16]. In particular, enhanced quantum fluctuations, leading to a crossover to a quantum spin glass, were evidenced in an applied transverse field [17].

In this Letter, we investigate a single crystalline $0.2 \%$ holmium doped $\mathrm{LiYF}_{4}$ at subkelvin temperatures. The isolated magnetic moments are weakly coupled by dipolar interactions $\left(\mu_{0} H_{\text {dip }} \sim\right.$ few $\left.\mathrm{mT}\right)$ so that this very diluted insulator should exhibit a nearly single ion quantum behavior, in which we are interested in continuity with our studies on molecular magnets. The crystal has a tetragonal scheelite structure with a $\mathrm{C}_{4 h}$ space symmetry group $\left(I 4_{1} / a\right)$, and the point symmetry group at $\mathrm{Ho}^{3+}$ sites is $\mathrm{S}_{4}$, almost equivalent to $\mathrm{D}_{2 d}$ (for $\mathrm{LiHoF}_{4}$, unit cell parameters are $a=b=5.175 \AA$ and $c=10.74 \AA$ [18]). Because of a very strong spin-orbit coupling, each magnetic ion of ${ }^{165} \mathrm{Ho}$ is characterized by its $J=8$ ground state manifold $\left(g_{J}=5 / 4\right)$, split by crystal field effects. These last give rise to a large uniaxial magnetic anisotropy, i.e., a high energy barrier hindering the magnetic moment reversal. However, we will see that quantum fluctuations due to significant transverse anisotropy terms drastically reduce this barrier. This effect was very much weaker in $\mathrm{Mn}_{12}$-ac $(\approx 10 \%$ barrier reduction [8]). Using the $|J, M\rangle$ basis and $\mathrm{D}_{2 d}$ symmetry, the approximate Hamiltonian including hyperfine interaction writes

$$
H=H_{\text {crystal field }}+H_{\text {Zeeman }}+H_{\text {hyperfine }}
$$

with

$$
\begin{aligned}
H_{\text {crystal field }}= & \alpha_{J} B_{2}^{0} O_{2}^{0}+\beta_{J}\left(B_{4}^{0} O_{4}^{0}+B_{4}^{4} O_{4}^{4}\right) \\
& +\gamma_{J}\left(B_{6}^{0} O_{6}^{0}+B_{6}^{4} O_{6}^{4}\right), \\
H_{\text {Zeeman }}= & -g_{J} \mu_{\mathrm{B}} \vec{J} \cdot \vec{H}, \quad H_{\text {hyperfine }}=A_{J} \vec{J} \cdot \vec{I} .
\end{aligned}
$$


The $\alpha_{J}, \beta_{J}, \gamma_{J}$, and $O_{l}^{m}$ are Stevens' coefficients and equivalent operators [19]. Exact diagonalization of the 136-dimensional Hamiltonian (1) was performed, using a set of crystal field parameters obtained by high-resolution optical spectroscopy: $B_{2}^{0}=273.9 \mathrm{~K}, B_{4}^{0}=-97.7 \mathrm{~K}$, $B_{6}^{0}=-6.5 \mathrm{~K}, B_{4}^{4}=-1289.1 \mathrm{~K}, B_{6}^{4}=-631.6 \mathrm{~K}[11]$. $A_{J}$ was taken as a fitting parameter of the measured resonances (see below). $J$ mixing, Jahn-Teller effect, and nuclear quadrupole interaction are assumed to be negligible. We first show the results with $A_{J}=0$ in Fig. 1. The eigenstates transform as one of the four irreducible representations $\Gamma_{1,2,3,4}$ of the $S_{4}$ point group. Significant transverse crystal field terms $B_{4}^{4} O_{4}^{4}$ and $B_{6}^{4} O_{6}^{4}$ mix free ion states, with $\Delta M= \pm 4$, so that eigenvectors are linear combinations either of $| \pm 7\rangle,| \pm 3\rangle,|\mp 1\rangle$, and $|\mp 5\rangle$ for $\Gamma_{3,4} ;| \pm 6\rangle,| \pm 2\rangle,|\mp 2\rangle$, and $|\mp 6\rangle$ for $\Gamma_{2}$; or $| \pm 8\rangle$, $| \pm 4\rangle,|0\rangle,|\mp 4\rangle$, and $|\mp 8\rangle$ for $\Gamma_{1}$. In Fig. 1(a), the calculated low-lying states within the ${ }^{5} I_{8}$ multiplet show a $\Gamma_{34}$ Ising ground state doublet noted as $\left|\psi_{1}^{ \pm}\right\rangle$, while the first excited state, a $\Gamma_{2}$ singlet, stands at $\approx 9.5 \mathrm{~K}$ above (direct measurements give $\approx 10 \pm 1 \mathrm{~K}$ [15]). Figure 1(a) also shows how the expected large barrier $\sim 10^{2} \mathrm{~K}$ is shortcut by large tunneling gaps due to transverse crystal field terms (emphasized by shaded areas between singlets belonging to the same representation). A strong electronic level repulsion in the low-lying excited $\Gamma_{2}$ states is clearly shown in Fig. 1(b). This defines the energy barrier the magnetic moment has to overcome in order to reverse its polarization. At very low temperatures, the system should be equivalent to a two-level system with an energy barrier of $\approx 10 \mathrm{~K}$. Actually, this picture is strongly modified when intraionic dipolar interactions are taken into account $\left(A_{J} \neq 0\right)$. They lead to a more complex diagram in the electronic ground state, showing several level crossings
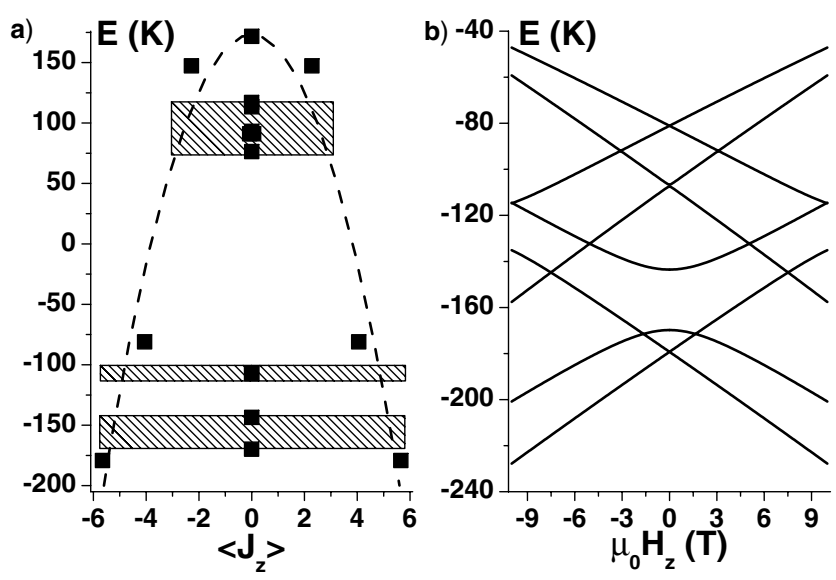

FIG. 1. (a) Energy levels vs average value of $J_{z}$, in zero applied field, showing an Ising ground state doublet and a first excited singlet at $\approx 9.5 \mathrm{~K}$ above. (b) Low-energy part of the Zeeman diagram. The first excited state $(\approx 25 \mathrm{~K}$ below the next excited $\Gamma_{2}$ singlet) defines an energy barrier of $\approx 9.5 \mathrm{~K}$ hindering the magnetic moment reversal. for resonant values $H_{n}(-7 \leq n \leq 7)$ in Fig. 2. The transverse hyperfine contribution $\frac{1}{2} A_{J}\left(J_{+} I_{-}+J_{-} I_{+}\right)$ induces some avoided level crossings between $\left|\psi_{1}^{-}, I_{z 1}\right\rangle$ and $\left|\psi_{1}^{+}, I_{z 2}\right\rangle$, with $\Delta I=\left|I_{z 2}-I_{z 1}\right|$, only when $\Delta I / 2$ is an odd integer so that the two electronic low-lying states $\left|\psi_{1}^{ \pm}\right\rangle$are coupled through nondegenerated excited electronic levels.

Magnetic measurements were made at $0.04<T<$ $1 \mathrm{~K}$ and for $\mu_{0} H<2 \mathrm{~T}$, with a micro-SQUID magnetometer [20] allowing field sweep rates up to $1 \mathrm{~T} / \mathrm{s}$. The crystal is first saturated in a large positive field applied along the $c$ axis $\mu_{0} H_{\text {sat }} \approx 0.3 \mathrm{~T}$, and then the field $H_{z}$ is swept between $\pm H_{\text {sat }}$. At slow field sweep rates, an isothermal process occurs, leading to staircaselike hysteresis loops at $T \leq 200 \mathrm{mK}$, as shown in Fig. 3 . These well defined steps come from quantum relaxation at avoided level crossings. At $T=50 \mathrm{mK}$, the lowest energy level is mainly populated and magnetization steps are observed for $-1 \leq n \leq 3$. The ground and excited tunnel splittings at $H_{z}=0$, being due to very small perturbations such as internal transverse fields, should be very similar. Therefore, quantum tunneling in zero field is mainly associated with the dynamics of the lowest avoided level crossing (the first excited crossing is at $\Delta E / k_{\mathrm{B}}=g_{\text {eff }} \mu_{\mathrm{B}} H_{1} / k_{\mathrm{B}} \approx 205 \mathrm{mK}$, assuming $g_{\text {eff }} \approx 13.3$ [14]). The amplitude of the next step, the resonance $n=1$ at $\mu_{0} H_{1}=23 \mathrm{mT}$, is about 10 times larger, suggesting a larger tunnel splitting $\Delta$. Indeed, Fig. 2 shows that the hyperfine induced tunnel splitting of the third excited avoided level crossing is large enough to render the barrier transparent $(\Delta \approx 25 \mathrm{mK})$. The relaxation time is thus simply given by thermal activation $\tau=\tau_{0} \exp \left(2 \Delta E / k_{\mathrm{B}} T\right)$, with a long $\tau_{0}$ because

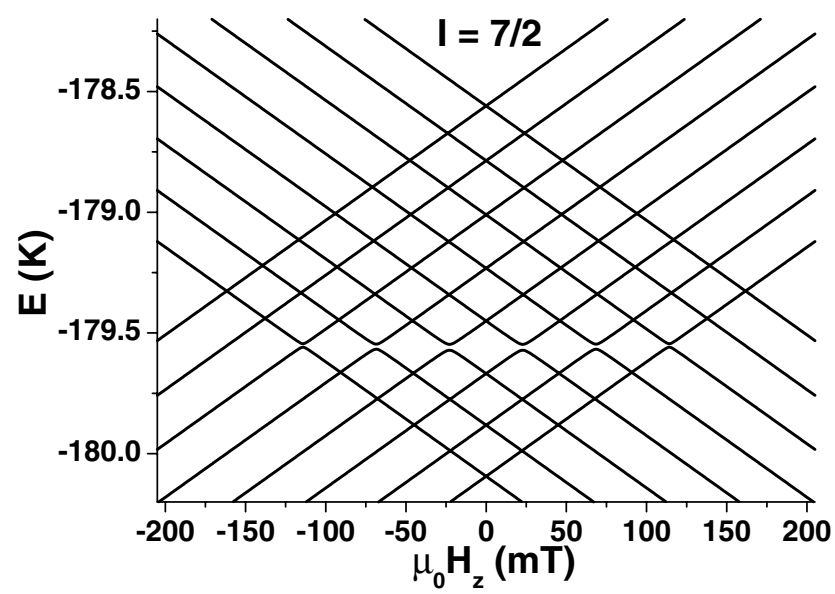

FIG. 2. Splitting of the electronic ground state doublet by the hyperfine interaction $\left(A_{J} / k_{\mathrm{B}} \approx 38.6 \mathrm{mK}\right.$; see below). The level crossings occur for resonant values of the longitudinal field $H_{n}$ $(-7 \leq n \leq 7)$. Some are avoided level crossings and hyperfine levels repulsion is then induced by the electronic level repulsion in the excited states shown in Fig. 1(b). 


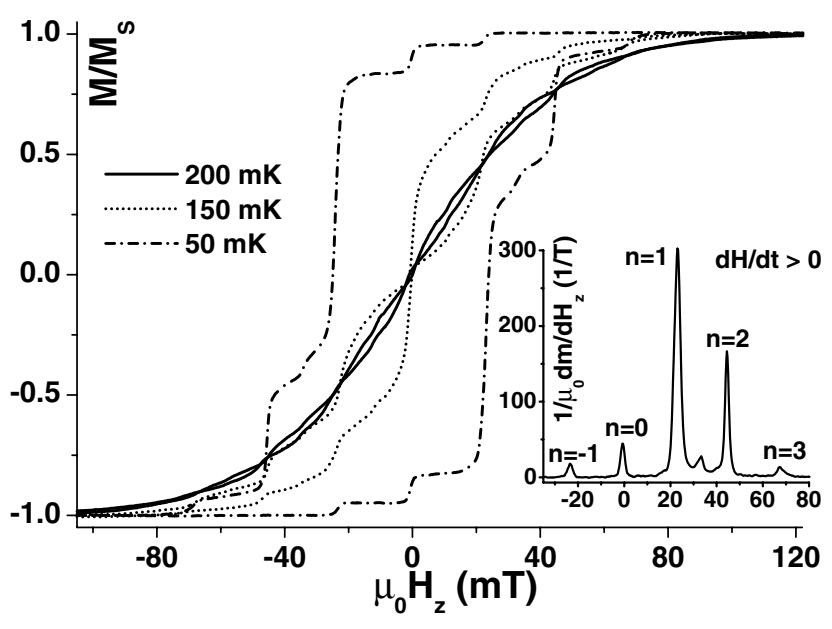

FIG. 3. Hysteresis loops for $v=0.55 \mathrm{mT} / \mathrm{s}$ and at different temperatures. Inset: derivative of the magnetization normalized to $M_{\mathrm{S}}, d m / d H$, at $T=50 \mathrm{mK}$ and for $v=0.55 \mathrm{mT} / \mathrm{s}$.

spin-lattice relaxation time $T_{1}$ can be hours at very low temperatures and/or as a result of internal fields fluctuations. In the same trend, the measured magnetization step ratio $\Delta M(n=1) / \Delta M(n=-1) \approx 25$ at $T=50 \mathrm{mK}$ is approximately equal to the Boltzmann ratio, which confirms that thermally activated quantum relaxation occurs at $H_{z} \neq 0$. The barrier, essentially transparent due to this large splitting, becomes again finite out of resonance. The quantum relaxation is strongly enhanced by a constant transverse field, as a result of an increase of the tunnel splittings (see Fig. 4). In zero longitudinal field, the small tunnel splittings rapidly increase and hysteresis vanishes. A saturation of the magnetization at $M \approx 0$ is

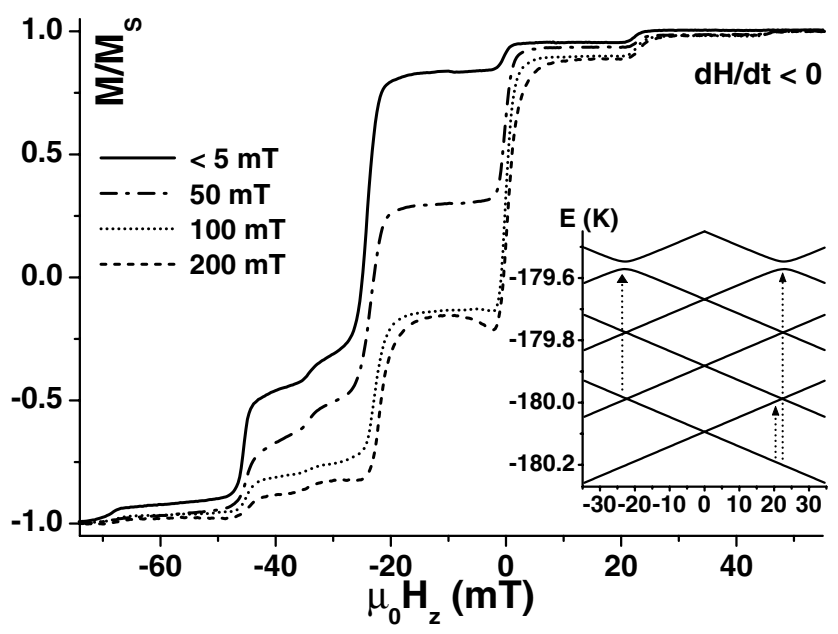

FIG. 4. Hysteresis loops in a constant transverse field at $T=$ $50 \mathrm{mK}$ and for $v=0.55 \mathrm{mT} / \mathrm{s}$. A transverse field enhances the quantum fluctuations in zero longitudinal applied field leading to a larger magnetization step. Inset: details of the Zeeman diagram around zero field. Thermally activated tunneling shows two possible channels over the first and the third, more efficient, excited avoided level crossings. observed in transverse fields larger than $100 \mathrm{mT}$, when the barrier is nearly transparent, and the small "overshot" with an oscillation in $M$ may be due to spin-phonon transitions. As expected for a large tunnel splitting, sensitivity to a small transverse field is very weak for the resonance $n=-1$. The inset in Fig. 3 shows $d m / d H$ at $T=50 \mathrm{mK}$. The width of the resonant transitions is about $\mu_{0} \Delta H=2-3 \mathrm{mT}$ which is expected from dipolar broadening. Similarly to molecular magnets, quasistatic fields due to dipolar interactions lead to a distribution of internal fields whereas field fluctuations, essentially of $\mathrm{F}^{-}$ nuclear spins, give homogeneous level broadening.

A hysteresis loop measured at $T=50 \mathrm{mK}$ for a much faster field sweep rate $(v=0.3 \mathrm{~T} / \mathrm{s})$ is shown in Fig. 5(a). A succession of equally spaced large and weak magnetization steps occur at fields $H_{n}$, with $-14 \leq 2 n \leq 14$. The larger ones, with integer $n$, are associated with several equally spaced level crossings and the smaller steps, with half integer $n$, fall just in between when the levels are equally spaced (see Fig. 2). $d m / d H$ is used to

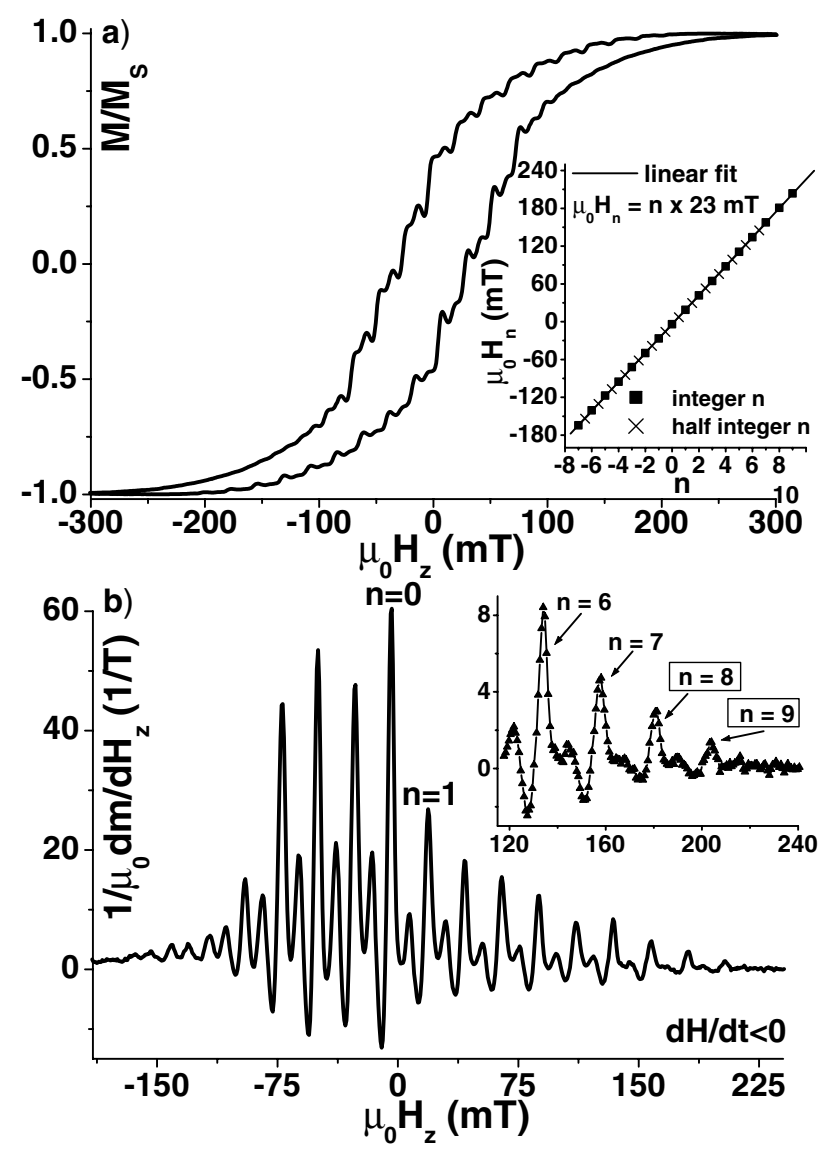

FIG. 5. (a) Hysteresis loops at $T=50 \mathrm{mK}$ and for $v=$ $0.3 \mathrm{~T} / \mathrm{s}$. Several magnetization steps are observed for resonant values of the applied field $\mu_{0} H_{n} \approx n \times 23 \mathrm{mT}$ (see inset; $H_{n}$ values are deduced from (b). (b) Derivative of the loop shown in (a) for a decreasing field. The two additional measured steps shown in the inset, for $n=8$ and $n=9$, are associated with cross-spin relaxation only. 
determine the $H_{n}$ values plotted in Fig. 5(a) inset. From the slope, we accurately obtain $\mu_{0} H_{n}=n \times 23 \mathrm{mT}$. The electronic ground doublet is thus split by hyperfine interaction in eight doublets over an energy range of about $1.44 \mathrm{~K}$. We deduce $A_{J} / k_{\mathrm{B}} \approx 38.62 \mathrm{mK}$, to be compared to $A_{J} / k_{\mathrm{B}} \approx 40.95 \mathrm{mK}$ [14]. The observed hysteresis loops depend sensitively on sample thermalization, showing that the spin-phonon system is not at equilibrium with the cryostat, leading to a phonon bottleneck $[10,21,22]$. At a fast field sweep rate $v=0.3 \mathrm{~T} / \mathrm{s}$, the system enters such a regime at $T \approx 1 \mathrm{~K}$ (moderate sample thermalization) showing hysteresis without any magnetization steps down to $T \approx 600 \mathrm{mK}$. When the field is swept back and forth, a stationary regime occurs and hysteresis loops become nearly temperature independent below a temperature $T_{c}(v)$ depending on sample thermalization $\left(T_{c} \approx 200 \mathrm{mK}\right.$ for $v=0.3 \mathrm{~T} / \mathrm{s}$ ). Below $T \approx 600 \mathrm{mK}$, a nearly adiabatic process occurs, due to a much longer spin-lattice relaxation time $T_{1}$. The spin bath becomes more and more isolated from the phonon bath and energy exchange between electronic and nuclear spins is possible only at fields $H_{n}$. Equilibrium within the spin system is due to either quantum fluctuations at avoided level crossings (integer $n$ ) or spin-phonon transitions and/or cross-spin relaxation, allowed by weak dipolar interactions, when energy levels are almost equally spaced (integer and half integer $n$ ) [22,23]. Spin-spin interactions allow two additional steps for $n=8$ and $n=9$, at fields with equally spaced levels but no level crossing [Fig. 5(b) inset]. A small transverse applied field increases only the zero-field magnetization step, showing the weak effect of enhanced quantum fluctuations on hysteresis loops in this regime. Other resonances and small magnetization steps, dominated by cross-spin relaxation, are not affected by a small transverse field, if small enough $\left(\mu_{0} H_{T} \lesssim 20 \mathrm{mT}\right)$. If the field sweep is suddenly stopped, the spin-phonon system exchanges energy with the cryostat and the magnetization relaxes toward the equilibrium curve. The asymmetry of the envelope (not drawn) of the peaks in Fig. 5(b), showing that spins reverse mostly after field inversion, and the absence of constriction in the hysteresis loop near $H_{z}=0$ confirm the existence of small barriers, mainly in zero field (tunneling).

In conclusion, we have shown that the quantum rotation of weakly coupled magnetic moments in $\mathrm{LiY}_{0.998^{-}}$ $\mathrm{Ho}_{0.002} \mathrm{~F}_{4}$ can be driven and monitored by hyperfine couplings. At very low temperatures, when the field is slowly swept from negative to positive values, the coupled electronic and nuclear moments tunnel from $\left|\psi_{1}^{-}, I_{z 1}\right\rangle$ to $\left|\psi_{1}^{+}, I_{z 2}\right\rangle$. In a constant transverse field, the magnetization step, associated with incoherent tunneling at the avoided level crossing in zero field, increases very rapidly. It saturates when the barrier is completely transparent. Details of hysteresis loops are in excellent agreement with the level structure of the electronic ground state doublet split by hyperfine interaction in 16 states. At faster field sweep rates, additional magnetization steps are observed and attributed to cross-spin relaxation and/or spin-phonon transitions in a phonon bottleneck regime. Very diluted holmium doped $\mathrm{LiYF}_{4}$ is thus a model system to study tunneling of an electronic moment strongly coupled to its nuclear spin.

We are very grateful to I. Chiorescu, J.C. Vial, and A. K. Zvezdin for discussions and to M.F. Joubert and P. Lejay for on-going collaborations. This work has been supported by DRET, Rhône-Alpes, MASSDOTS ESPRIT, MolNanoMag TMR, and AFIRST.

[1] A. J. Leggett et al., Rev. Mod. Phys. 59, 1 (1987).

[2] B. Barbara et al., J. Magn. Magn. Mater. 140-144, 1825 (1995); A. Novak and R. Sessoli, in Quantum Tunneling of Magnetization, edited by L. Gunther and B. Barbara, NATO ASI, Ser. E, Vol. 301 (Kluwer, Dordrecht, 1995), p. 171.

[3] L. Thomas et al., Nature (London) 383, 145 (1996); J. R. Friedman et al., Phys. Rev. Lett. 76, 3830 (1996).

[4] C. Sangregorio et al., Phys. Rev. Lett. 78, 4645 (1997); W. Wernsdorfer and R. Sessoli, Science 284, 133 (1999).

[5] N. V. Prokof'ev and P. C. E. Stamp, J. Phys. Condens. Matter 5, L663 (1993); J. Low Temp. Phys. 104, 143 (1996); A. Garg, Phys. Rev. Lett. 74, 1458 (1995).

[6] N. V. Prokof'ev and P. C. E. Stamp, Rep. Prog. Phys. 63, 669 (2000).

[7] W. Wernsdorfer et al., Phys. Rev. Lett. 84, 2965 (2000).

[8] B. Barbara et al., J. Magn. Magn. Mater. 200, 167 (1999).

[9] I. Tupitsyn and B. Barbara, in Magnetoscience-From Molecules to Materials, edited by J.S. Miller and M. Drillon (Wiley VCH, Weinheim, 2000).

[10] I. Chiorescu et al., Phys. Rev. Lett. 84, 3454 (2000).

[11] Sh. N. Gifeisman et al., Opt. Spectrosc. (USSR) 44, 68 (1978); N. I. Agladze et al., Phys. Rev. Lett. 66, 477 (1991).

[12] N. Vernier and G. Bellessa, J. Magn. Magn. Mater. 177-181, 962 (1998).

[13] P.E. Hansen et al., Phys. Rev. B 12, 5315 (1975); P. Beauvillain et al., ibid. 18, 3360 (1978); P. Beauvillain, Ph.D. thesis, Université Paris-Sud, 1979.

[14] J. Magariño et al., Phys. Rev. B 13, 2805 (1976).

[15] G. Mennenga et al., J. Magn. Magn. Mater. 44, 59 (1984).

[16] D. H. Reich et al., Phys. Rev. B 42, 4631 (1990).

[17] W. Wu et al., Phys. Rev. Lett. 67, 2076 (1991).

[18] E. R. Thoma et al., J. Phys. Chem. 65, 1906 (1961).

[19] K. W. H. Stevens, Proc. Phys. Soc. London Sect. A 65, 209 (1952).

[20] W. Wernsdorfer et al., Phys. Rev. Lett. 79, 4014 (1997).

[21] J. H. Van Vleck, Phys. Rev. 59, 724 (1941); A. Abragam and B. Bleaney, Electronic Paramagnetic Resonance of Transition Ions (Clarendon Press, Oxford, 1970); P. L. Scott and C. D. Jeffries, Phys. Rev. 127, 32 (1962).

[22] J. C. Verstelle and D. A. Curtis, in Handbuch Der Physik, Magnetismus (Springer-Verlag, Berlin, 1968).

[23] N. Bloembergen et al., Phys. Rev. 114, 445 (1959); K. H. Hellwege et al., Z. Phys. 217, 373 (1968). 